\title{
Can a brief psychological intervention improve oral health behaviour? A randomised controlled trial
}

\author{
U. Wide ${ }^{*}$, J. Hagman, H. Werner and M. Hakeberg
}

\begin{abstract}
Background: Dental caries is a major public health issue affecting a large proportion of the general population. The disease is associated with behavioural factors and is thus preventable to a high degree. Individuals may need assistance to be able to change their oral health behaviour. There is a lack of such interventions for adults affected by severe caries. The aim of the study was to evaluate the effect of Acceptance and Commitment Therapy (ACT), a form of cognitive behavioural therapy, on oral health behaviour in young adults with poor oral health.

Methods: The study included a two group parallel randomised controlled trial at general dental clinics, with young adults, $18-25$ years of age, $\geq$ two manifest proximal dental caries lesions $(n=135) ; 67$ were treated with ACT and 68 with standard disease information only, respectively. Primary outcomes: oral health behaviours (tooth-brushing, flossing, use of toothpicks, and additional fluoride use). The CONSORT principles for RCTs were used, including intention-to-treat and per protocol analyses. The Chi-square, Mann-Whitney, and Wilcoxon Signed Rank tests were applied, including effect sizes.
\end{abstract}

Results: The study groups did not differ with regard to oral health behaviour variables at baseline. The intervention group improved all their oral health behaviours significantly over time (effect sizes, $0.26-0.32$ ), while the control group showed improved behaviours on two measures (flossing and additional use of fluoride, effect sizes, 0.22-0. 23).

Conclusions: By testing a psychological intervention on young adults (18-25 years of age) with a high prevalence of caries, we found an immediate positive effect with improved oral health behaviours.

Trial registration: TRN ISRCTN15009620, retrospectively registered 14/03/2018.

Keywords: Acceptance and commitment therapy, Cognitive behaviour therapy, Psychological intervention, Caries, Young adults, Oral health behaviours, Randomised controlled trial

\section{Background}

Dental caries is a major public health issue, affecting around $60-90 \%$ of children, adolescents and adults worldwide [1]. Dental caries is associated with negative consequences and costs to sufferers and oral care providers [2, 3]. Moreover, dental caries is largely related to behavioural factors, such as oral hygiene, fluoride exposure and dietary habits. Thus, dental caries may be

\footnotetext{
* Correspondence: ulla.wide@gu.se

Department of Behavioral and Community Dentistry, Institute of Odontology, The Sahlgrenska Academy, University of Gothenburg, P.O. Box 450, SE-40530 Gothenburg, Sweden
}

(c) The Author(s). 2018 Open Access This article is distributed under the terms of the Creative Commons Attribution 4.0 International License (http://creativecommons.org/licenses/by/4.0/), which permits unrestricted use, distribution, and reproduction in any medium, provided you give appropriate credit to the original author(s) and the source, provide a link to the Creative Commons license, and indicate if changes were made. The Creative Commons Public Domain Dedication waiver (http://creativecommons.org/publicdomain/zero/1.0/) applies to the data made available in this article, unless otherwise stated.

treated and prevented with behavioural interventions at the individual level.

Recent research in public health stresses the social determinants of oral health and inequalities in health, and the need for structural interventions to improve health and reduce health inequalities [4-6]. However, the dental care practice also needs effective methods to help individuals with poor oral health to change their behaviour.

One recent systematic review found evaluations and, to some degree, positive effects of behavioural interventions for adult individuals in the field of dentistry, mainly for older adults affected by periodontitis (besides 
caries, the other major oral disease) [7]. Similar findings were reported by Newton and Asimakopoulou for behaviour interventions in improving oral hygiene related behaviour in patients with periodontitis [8]. However, a systematic review found no behavioural interventions for dietary change in adult patients with dental caries [9]. Thus, less is known about behavioural interventions for adults affected by caries. Young adults develop health behaviour habits for their adult lives, and possibly for their children, and are therefore an important group to target for behavioural interventions. Authors have emphasised the need to use stringent interventions based on accepted theory from the behavioural sciences (the field of health psychology) to affect oral health behaviour changes $[10,11]$.

Different theory-based interventions for behaviour change has been developed, and to some degree tested for oral health problems. The present study was designed to evaluate a brief psychological intervention, Acceptance and commitment therapy (ACT), delivered by a psychologist in general dentistry, as a means to help young adult patients with poor oral health to make behavioural changes to improve their oral health.

ACT is a recently developed psychological method [12], a form of Cognitive Behaviour Therapy (CBT), that has been used with positive results in the treatment of health problems, such as pain, tinnitus and addiction $[13,14]$. ACT interventions have been developed in brief formats for primary care, a setting similar to general dentistry, but has, to our knowledge, not been tested in general dentistry. The rationale of ACT is to increase psychological flexibility, thus facilitating the individual to maintain functional behaviours, and to change dysfunctional behaviours, in order to live in accordance with chosen individual life values [15]. The intention of using ACT in the present study was to contribute to behaviour change by focusing on how health-related behaviour could be relevant to valued life directions, by addressing also the psychological flexibility of the individual.

\section{Methods}

\section{The aim and design of the study}

The aim of the study was to evaluate the effect of ACT on oral health behaviours in young adults with poor oral health. Hypothesis: A brief psychological intervention (ACT) improves oral health behaviours (such as tooth-brushing and flossing) more than standard information alone.

The present analysis is part of a larger clinical trial evaluating the effect of ACT on oral health behaviours, oral health (caries, gingivitis), sugar consumption, psychological distress, general health behaviour and the ability to handle stress. In the present analysis the primary outcomes were oral health be haviours (flossing, toothpick use, tooth-brushing, ad ditional fluoride use).

The study was a two group parallel randomised controlled trial with an allocation ratio 1:1. The study was approved by the Regional Ethical Review Board in Gothenburg (Reg. no. 840-12).

\section{Participants}

The participants were recruited between 2013 and 2014 at two Public Dental Service clinics in Region Västra Götaland, Sweden. Inclusion criteria: $18-25$ years of age, $\geq$ two manifest proximal dental caries lesions. Exclusion criteria: Psychiatric/neuropsychiatric diagnosis, such as depression, psychosis, autism spectrum disorder, mental retardation, substance abuse. Participants needed to have good understanding of Swedish which was assessed by the research coordinator. A power analysis was performed to determine the sample size. The calculation was made for gingivitis (mean ratio of bleeding surfaces) and the assumption of detecting a $20 \%$ reduction with an alpha of 0.05 and a power of 0.80 . The number of participants needed was 53 individuals per group. Thus, including dropouts, the sample was determined to require at least 130 participants, 65 per group. Power calculations were repeated with other outcome variables (plaque, caries, oral health behaviours), but these did not change the minimum number of participants needed to detect a relevant difference between groups.

Potentially eligible individuals were screened (first screening) while at their ordinary routine dental examination, and were invited to participate in the trial. The research coordinator at the dental clinic contacted individuals interested in participating, and after a second screening/confirmation of the inclusion/exclusion criteria, the individual received written information about the trial. The study participants were asked for and provided written consent. The second screening resulted in 186 eligible patients. Of these, 51 declined to participate, the most common reasons being "not interested" and "lack of time" (see Fig. 1). The final sample consisted of 135 participants (acceptance rate $72.6 \%$ ).

\section{Procedure and allocation strategy}

Individuals included in the study answered baseline questionnaire using a touch-screen computer. Clinical data were obtained from their most recent ordinary dental examination. All participants then received standardised oral health information, provided verbally by a registered dental nurse using a brochure on oral health behaviour and caries. The information, including the brochure, was at the time of the study used at all public dental service clinics in Region Västra Götaland, Sweden.

The participants were then randomised by an independent research coordinator, either to the Intervention 


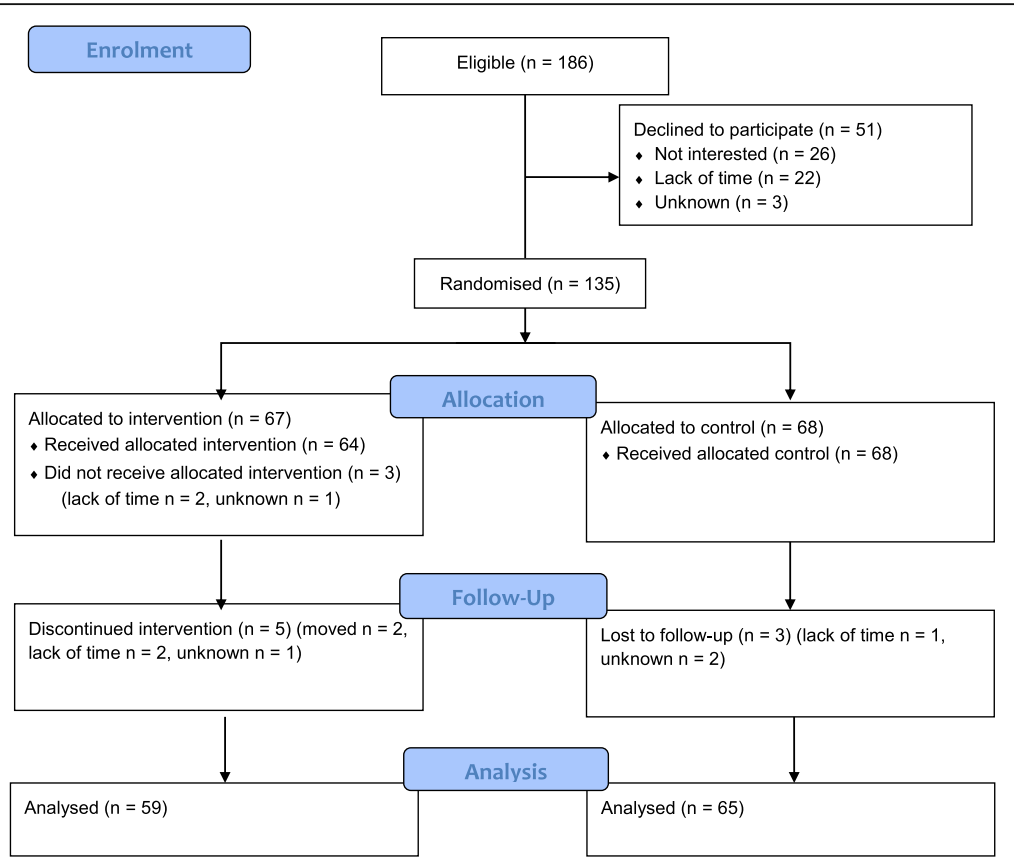

Fig. 1 Flow diagram of the progress through the phases of the Intervention group and Control group: Enrolment, intervention allocation, follow-up and data analyses

(ACT plus information) or Control (information alone) group, using a block randomisation procedure including stratification by gender and smoking (randomly permuted blocks within strata [16]). As an allocation strategy, the research coordinator used sealed opaque envelopes that had been prepared in advance by another research coordinator and placed in four boxes according to the stratification strategy. The allocation list was kept in a locked safety box, only available to the independent research coordinator.

Participants allocated to intervention were scheduled for two appointments with the psychologist 2 weeks apart (see below for description of the intervention). Participants answered follow-up questions at the clinic 3 weeks after baseline. (See Fig. 1, Flow chart according to CONSORT [17]).

At the two dental clinics, the study involved general practitioners (dentists and dental hygienists), a research coordinator, dental nurses and a clinical psychologist. All treatments and examinations were performed at the respective clinic.

\section{Measures}

Clinical measure of oral health: data on dental caries lesions (number and type) according to accepted standards (D1-D3, secondary caries), including assessment of caries on four surfaces, with proximal caries assessed on radiographs [18]. A summarised score of the number of surfaces with manifest caries (D3 and secondary caries) was calculated.
Sociodemographic characteristics were measured with questions about: age, gender, ethnicity (Swedish-born, including other Nordic country; foreign-born), mother's country of birth (Swedish-born, including other Nordic country; foreign-born), housing (rented flat; own flat/ house; other), mother's education (primary; secondary; university).

Self-rated oral health was captured with the question 'How do you rate your oral health?', with four response alternatives (poor; fair; good; very good).

Oral health behaviour was assessed with questions about tooth-brushing, flossing, use of toothpicks, and use of additional fluoride (besides toothpaste), with six response alternatives: three times a day or more; twice a day; once a day; several times a week; once a week; more seldom/never. One question measured dental care attendance, where the five response alternatives were dichotomised into often (twice a year; once a year) vs. seldom (every other year; less then every other year; only when acute problem).

Any adverse effects during the study period reported by the participants were registered by the research coordinators.

\section{Intervention}

The intervention used was a psychological intervention, CBT in the ACT form $[12,15]$, adapted to primary care settings $[19,20]$ and modified for the present trial. The modification included a selection of well-known ACT exercises (e.g., defusion and Bull's Eye), and was made in 
close collaboration with a licensed psychologist specialised in $\mathrm{ACT}$ and experienced in implementing $\mathrm{ACT}$ in primary care. Like other CBT-interventions, ACT is based on an individual case conceptualisation and a functional analysis of behaviour, and the participant and the psychologist together develop a plan for behaviour change. In Table 1 a treatment overview is provided, showing the different ACT modules.

The intervention was delivered at two general dental clinics and included two individual sessions (45 $\mathrm{min}$ each) with a licensed psychologist specialised in ACT (HW). To secure adherence to treatment, the psychologist in the project was regularly supervised [21]. The time between the first and the second session was 2 weeks.

\section{Statistical analyses}

Descriptive statistics used were frequencies, mean, median and standard deviation (SD). The statistical methods applied were the Chi-square test, the Mann-Whitney test for independent groups, and the Wilcoxon Signed Rank test for dependent groups. Both intention-to-treat (ITT) and per protocol (PP) analyses were performed according to the CONSORT principles [17]. The effect size according to Cohen's ES was calculated for changes over time, applying the Wilcoxon Signed Rank test using the formula $\mathrm{z} /$ $\sqrt{ } N$, where $\mathrm{z}$ is the test statistic and $\mathrm{N}$ equals twice the number of individuals included in the respective analyses [22]. According to Cohen's criteria [23], an effect size around $0.1=$ low effect, $0.3=$ medium effect, and $0.5=$ large effect. The significance level applied was 0.05. Bonferroni corrections for multiple comparisons were applied giving $p$-values for statistical significance of $p<0.005$ for baseline (Table 2), and $p<0.003$ for primary outcomes (Table 3).. The study included a blinded design with the research group and statistical analyst being blinded to which treatment was allocated to which patient.

Table 1 Treatment overview of ACT for patients with dental caries

\begin{tabular}{ll}
\hline Session 1 & Session 2 \\
\hline Introduction & Follow-up \\
Brief interview & Bull's-Eye \\
Mindful oral health & Mindful oral health \\
Focused questions & Value based living \\
Case conceptualisation & Defusion exercises \\
Bull's-Eye & Plan for behavioural change and follow-up \\
Clarification of values & \\
Plan for behavioural change & \\
\hline
\end{tabular}

\section{Results}

\section{Description of participants at baseline}

In total, 135 individuals were included in the study, and were allocated to either intervention $(n=67)$ or control $(n=68)$. Sociodemographic and clinical characteristics of the participants are presented in Table 2. The mean age was 20 years, and the participants had a mean number of caries surfaces of 6.3 and 4.9 in the intervention and control group, respectively. The vast majority of participants experienced their oral health to be poor or fair, with $86.6 \%$ and $82.4 \%$ in the intervention and control group, respectively. About $40 \%$ in both groups rated their oral health as poor, less than $20 \%$ rated their oral health as good, and none rated it as very good. Half of the subjects were female, one third was smokers, and different ethnicities and socioeconomic positions were represented in the study group. The intervention group reported statistically significantly more Swedish-born mothers than the control group $(65.7 \%$ vs. $42.6 \%, p<$ 0.01 (ns. after Bonferroni correction)), while the groups did not differ with regard to the other sociodemographic and clinical measures. The study groups did not differ with regard to oral health behaviour variables at baseline (Table 3).

\section{Changes after intervention}

The number of participants who received intended treatment and were analysed, as well as participant losses after randomization, are presented in Fig. 1. In the intervention group, 64 individuals received the allocated treatment and 59 of them participated in the follow-up, while 68 individuals received the control condition, and 65 of them participated in the follow-up. Per protocol analyses revealed that the intervention group improved their oral health behaviour on all four measures (Table $3)$ : tooth-brushing $(Z=-3.43, p=0.001$, effect size 0.32$)$; flossing $(Z=-3.48, p=0.0005$, effect size 0.32$)$; toothpicks $(Z=-3.04 p=0.002$, effect size 0.28); additional use of fluoride $(Z=-3.27 p=0.001$, effect size 0.30$)$. The control group improved their oral health behaviour regarding two variables: use of flossing $(\mathrm{Z}=-2.72, p=$ 0.006 (ns. after Bonferroni correction), effect size 0.24) and additional use of fluoride $(Z=-2.53, p=0.011$ (ns. after Bonferroni correction), effect size 0.22 ), while no differences were found regarding tooth-brushing $(\mathrm{Z}=-$ $0.99, p=0.320)$ and toothpicks $(Z=-0.73, p=0.466)$.

Intention-to-treat analyses showed parallel results in that the intervention group improved their oral health behaviour on all four measures (Table 3): tooth-brushing $(\mathrm{Z}=-3.43, p=0.001$, effect size 0.30$)$; flossing $(\mathrm{Z}=-$ $3.48, p=0.0005$, effect size 0.30$)$; toothpicks $(Z=-3.04$ $p=0.002$, effect size 0.26 ); additional use of fluoride ( $\mathrm{Z}$ $=-3.27 \mathrm{p}=0.001$, effect size 0.28 ). The control group improved their oral health behaviour over time 
Table 2 Sociodemographic and clinical characteristics of participants $(n=135)$ allocated to Intervention or Control, at baseline

\begin{tabular}{|c|c|c|c|}
\hline Variable & Intervention $(n=67)$ & Control $(n=68)$ & $\mathrm{P}$ \\
\hline Age in years, Mean (SD) & $20.4(2.1)$ & $20.8(2.2)$ & ns. \\
\hline Self-rated oral health, $\mathrm{n}(\%)$ & & & ns. \\
\hline Poor & $27(40.3)$ & $25(36.8)$ & \\
\hline Fair & $31(46.3)$ & $31(45.6)$ & \\
\hline Good & $9(13.4)$ & $12(17.6)$ & \\
\hline Very good & 0 & 0 & \\
\hline Caries, Mean (SD) Median & $6.3(5.2) 4$ & $4.9(3.7) 5$ & ns. \\
\hline Dental care attendance, $\mathrm{n}(\%)$ often & $58(86.6)$ & $56(82.4)$ & ns. \\
\hline Gender, n (\%) female & $32(47.8)$ & $32(47.1)$ & ns. \\
\hline Smoker, n (\%) smoking & $23(34.3)$ & $24(35.3)$ & ns. \\
\hline Ethnicity, n (\%) Swedish-born & $55(82.1)$ & $48(70.6)$ & ns. \\
\hline Housing, n (\%) & & & ns. \\
\hline Rental flat & $32(47.8)$ & $33(48.5)$ & \\
\hline Own flat/house & $28(41.8)$ & $25(36.8)$ & \\
\hline Other & $7(10.4)$ & $10(14.7)$ & \\
\hline Mother's ethnicity, n (\%) Swedish-born & $44(65.7)$ & $29(42.6)$ & $p<0.01^{a}$ \\
\hline Mother's education, n (\%) & & & ns. \\
\hline Primary & $15(22.4)$ & $22(32.4)$ & \\
\hline Secondary & $35(52.2)$ & $31(45.6)$ & \\
\hline University & $17(25.4)$ & $15(22.1)$ & \\
\hline
\end{tabular}

Chi-square (Mann-Whitney for caries), ${ }^{a}$ Ns after Bonferroni correction

concerning two variables: use of flossing $(\mathrm{Z}=-2.72, p=$ 0.006 (ns. after Bonferroni correction), effect size 0.23 ), and additional use of fluoride $(\mathrm{Z}=-2.53, p=0.011$ (ns. after Bonferroni correction), effect size 0.22), while no differences were found concerning tooth-brushing $(\mathrm{Z}=$ $-0.99, p=0.320)$ and toothpicks $(\mathrm{Z}=-0.73, p=0.466)$.

No adverse events were reported by the participants. The period of recruitment of participants to final examination at follow-up lasted between February 2013 and May 2016.

\section{Discussion}

This randomised controlled trial evaluated the effect of a brief psychological intervention (ACT) for behaviour change, delivered by a psychologist in general dental care to young adults (18-25 years of age) with dental caries. Significant positive changes with regard to oral health behaviours were found, most prominent in the intervention group compared with the control group that received standardised information. However, the hypothesis stated was only accepted in part regarding the measures of oral health behaviours (i.e., the ACT intervention improved oral health behaviours more than information alone).

There are mixed results in the literature on behavioural interventions to improve oral health behaviour in individuals with poor oral health $[7,24]$. Positive effects on tooth-brushing and interdental cleaning have been reported in studies with an RCT design including middle-aged to older individuals with periodontitis [2527]. In the present study on young adults (18-25 years of age) with dental caries, the intervention group improved their oral health behaviour on all investigated variables (tooth-brushing, flossing, use of toothpicks and additional use of fluoride). This is a promising result. The participants in this study were all affected by severe dental caries disease, and behavioural change was necessary to halt the disease progression and to promote better oral health.

The control group also showed some improvement in oral health behaviour, although on fewer measures. There are potential general effects of being a participant in a clinical study, such as receiving extra attention from dental personnel, which may contribute to positive changes also in the control group. It is not reasonable to argue that the control condition in itself led to these changes in the control group, since the control condition consisted of the ordinary treatment-as-usual information delivered to all patients.

Previous studies on psychological interventions for behavioural change in the area of dentistry have mainly focused on interventions inspired of or based on the Motivational Interviewing technique, applied to patients with periodontitis at specialised clinics [26, 28-30]. 
Table 3 Oral health behaviour of the participants allocated to Intervention or Control, at baseline and follow-up, according to Per Protocol (PP) and Intention-To-Treat (ITT) analyses, respectively

\begin{tabular}{|c|c|c|c|c|c|c|c|c|}
\hline \multirow[t]{3}{*}{ Variable } & \multicolumn{4}{|l|}{ Baseline } & \multicolumn{4}{|l|}{ Follow-up } \\
\hline & \multicolumn{2}{|l|}{ Intervention } & \multicolumn{2}{|l|}{ Control } & \multicolumn{2}{|l|}{ Intervention } & \multicolumn{2}{|l|}{ Control } \\
\hline & $\mathrm{ITT}(n=67)$ & $\mathrm{PP}(n=59)$ & $\mathrm{ITT}(n=68)$ & $\mathrm{PP}(n=65)$ & $\mathrm{ITT}(n=67)$ & $\mathrm{PP}(n=59)$ & $\mathrm{ITT}(n=68)$ & $\mathrm{PP}(n=65)$ \\
\hline \multicolumn{9}{|l|}{ Tooth-brushing } \\
\hline$\geq 3$ times/day & $1(1.5)$ & $1(1.7)$ & $2(2.9)$ & $2(3.1)$ & $2(3.0)$ & $2(3.4)$ & $3(4.4)$ & $3(4.6)$ \\
\hline Twice a day & $37(55.2)$ & $32(54.2)$ & $43(63.2)$ & $42(64.6)$ & $50(74.6)$ & $45(76.3)$ & $46(67.6)$ & $45(69.2)$ \\
\hline Once a day & $16(23.9)$ & $14(23.7)$ & $13(19.1)$ & $12(18.5)$ & $8(11.9)$ & $6(10.2)$ & $9(13.2)$ & $8(12.3)$ \\
\hline Several times/week & $8(11.9)$ & $7(11.9)$ & $9(13.2)$ & $8(12.3)$ & $6(9.0)$ & $5(8.5)$ & $9(13.2)$ & $8(12.3)$ \\
\hline Once a week & $4(6.0)$ & $4(6.8)$ & $1(1.5)$ & $1(1.5)$ & $1(1.5)$ & $1(1.7)$ & $1(1.5)$ & $1(1.5)$ \\
\hline More seldom/never & $1(1.5)$ & $1(1.7)$ & 0 & 0 & 0 & 0 & 0 & 0 \\
\hline \multicolumn{9}{|l|}{ Flossing } \\
\hline$\geq 3$ times/day & 0 & 0 & 0 & 0 & $2(3.0)$ & $2(3.4)$ & $2(2.9)$ & $2(3.1)$ \\
\hline Twice a day & $7(10.4)$ & $6(10.2)$ & $5(7.4)$ & $5(7.7)$ & $13(19.4)$ & $12(20.3)$ & $7(10.3)$ & $7(10.8)$ \\
\hline Once a day & $4(6.0)$ & $4(6.8)$ & $10(14.7)$ & $10(15.4)$ & $11(16.4)$ & 11 (18.6) & 14 (20.6) & $14(21.5)$ \\
\hline Several times/week & $14(20.9)$ & $11(18.6)$ & $11(16.2)$ & $10(15.4)$ & $13(19.4)$ & $10(16.9)$ & $12(17.6)$ & $11(16.9)$ \\
\hline Once a week & $10(14.9)$ & $9(15.3)$ & $8(11.8)$ & $8(12.3)$ & $9(13.4)$ & $8(13.6)$ & $12(17.6)$ & $12(18.5)$ \\
\hline More seldom/never & $32(47.8)$ & $29(49.2)$ & $34(50.0)$ & $32(49.2)$ & $19(28.4)$ & $16(27.1)$ & $21(30.9)$ & 19 (29.9) \\
\hline \multicolumn{9}{|l|}{ Toothpick use } \\
\hline$\geq 3$ times/day & $1(1.5)$ & $1(1.7)$ & $1(1.5)$ & $1(1.5)$ & $3(4.5)$ & $3(5.1)$ & 0 & 0 \\
\hline Twice a day & $1(1.5)$ & $1(1.7)$ & $2(2.9)$ & $2(3.1)$ & $4(6.0)$ & $4(6.8)$ & $2(2.9)$ & $2(3.1)$ \\
\hline Once a day & $2(3.0)$ & $2(3.4)$ & $7(10.3)$ & $6(9.2)$ & $7(10.4)$ & $7(11.9)$ & $11(16.2)$ & $10(15.4)$ \\
\hline Several times/week & $6(9.0)$ & $6(10.2)$ & $7(10.3)$ & $7(10.8)$ & $5(7.5)$ & $5(8.5)$ & $6(8.8)$ & $6(9.2)$ \\
\hline Once a week & $4(6.0)$ & $3(5.1)$ & $3(4.4)$ & $2(3.1)$ & $5(7.5)$ & $4(6.8)$ & $6(8.8)$ & $5(7.7)$ \\
\hline More seldom/never & $53(79.1)$ & $46(78.0)$ & 48 (70.6) & $47(72.3)$ & $43(64.2)$ & $36(61.0)$ & $43(63.2)$ & $42(64.6)$ \\
\hline \multicolumn{9}{|l|}{ Additional fluoride } \\
\hline$\geq 3$ times/day & $3(4.5)$ & $3(5.1)$ & $4(5.9)$ & $3(4.6)$ & $5(7.5)$ & $5(8.5)$ & $3(4.4)$ & $2(3.1)$ \\
\hline 2 times/day & $13(19.4)$ & $11(18.6)$ & $8(11.8)$ & $7(10.8)$ & $19(28.4)$ & $17(28.8)$ & 14 (20.6) & $13(20.0)$ \\
\hline Once a day & $12(17.9)$ & $11(18.6)$ & $14(20.6)$ & $14(21.5)$ & $13(19.4)$ & $12(20.3)$ & $17(25.0)$ & $17(26.2)$ \\
\hline Several times/week & $10(14.9)$ & $8(13.6)$ & $13(19.1)$ & $13(20.0)$ & $16(23.9)$ & $14(23.7)$ & $18(26.5)$ & $18(27.7)$ \\
\hline Once a week & $10(14.9)$ & $9(15.3)$ & $10(14.7)$ & $10(15.4)$ & $4(6.0)$ & $3(5.1)$ & $6(8.8)$ & $6(9.2)$ \\
\hline More seldom/never & $19(28.4)$ & $17(28.8)$ & $19(27.9)$ & $18(27.7)$ & $10(14.9)$ & $8(13.6)$ & $10(14.7)$ & $9(13.8)$ \\
\hline
\end{tabular}

Frequency $\mathrm{n}(\%)$

Interventions based on other theoretical models have also been presented [27, 31, 32]. This study adds important knowledge to the field by testing ACT, a theory-based psychological intervention used with promising results for various health issues in health care $[13,14]$ but, to our knowledge, not previously used in dentistry. With regard to ACT, one may specifically emphasize certain modules in the first session, such as the brief interview and focused questions leading to the case conceptualisation, providing information and stance for individualised interventions to increase psychological flexibility and contact with life values. However, we believe that the all-embracing model of ACT as a specific type of CBT-intervention is the most important factor for the behaviour changes accomplished.
The present study also used a multi-professional setting, where dental personnel identified eligible participants, and where the intervention was delivered by a licensed psychologist working at the same general dental clinic. Over the last decades it has become more common to include psychologists in primary care settings [33]. The same development has not taken place within dentistry, with the exception of treatment of patients with dental phobia, where psychologists are members of treatment teams in many specialised clinics [34-36].

In this paper we have discussed the effect of ACT on oral health behaviour. Other behavioural outcomes of relevance for oral health are for example tobacco use and dietary habits. Behaviour change interventions or 
counselling has proven effective for tobacco use cessation in adults in both general medicine and dentistry [37]. However, according to a Cochrane review [38], there is limited evidence about effective interventions (behavioural and/or medical) for smoking cessation in young people. When it comes to dietary habits, there is evidence from the field of general medicine that behaviour change or counselling could effectively change such habits [37]. Yet, in dentistry, such interventions have only had limited effect. In fact, as mentioned previously, a recent systematic review on interventions for dietary change in adult patients with dental caries found no such studies [9]. Thus, there are several knowledge gaps to address in the future.

This study has some strengths and limitations. The study used an appropriate RCT design while adhering to the standard protocol for such a design, according to the CONSORT methodology. We included a large number of participants at baseline and had a dropout rate of only $15.5 \%$ at follow-up. Moreover, the analyses included both per protocol and intention-to-treat evaluations. The study group of young adults, between the ages of 18 and 25 years, is in a period of their lives when mobility is common. Individuals move away from home, find employment or may enrol in higher education; thus, an even greater loss to follow-up was expected. The generalisability of the study is high, as the study was conducted in two general Public Dental Service clinics. In Sweden, the large majority of individuals in this age-group regularly visit Public Dental Service clinics. The participants were recruited while on their ordinary visit at the clinics, where registrations and interventions were performed. A desirable double-blind procedure was obviously not possible, due to the design and intervention tested. However, we were able to blind the research group and the statistician to which group the participants belonged. The outcome measures are self-reported only and it is therefore important to include objective clinical health measurements, such as gingivitis and caries. Even if the results are promising with regard to oral health behaviour, we need to conclude on the long-term effects of the psychological intervention, i.e. the sustainability of the results.

To the best of our knowledge, while searching the scientific literature, we have not found other RCTs testing a behavioural intervention on young adults $(18-25$ years of age) with high caries activity, nor do we know of a similar field study setting where a licensed psychologist has been employed within general dentistry clinics to treat young adults affected by caries disease. It may be argued that the dental professions need other professionals, such as psychologists, when treating or counselling young adults in order to alter their health behaviour related to different oral diseases. Moreover, this is particularly important considering the often high prevalence of oral diseases, the close relationship between oral diseases and health behaviour, and the fact that these diseases, in terms of etiologic fraction, are highly preventable.

\section{Conclusions}

By testing a psychological intervention (Acceptance and Commitment Therapy) on young adults (18-25 years of age) with high caries prevalence, we found an immediate positive effect with improved oral health behaviours, including more tooth-brushing, flossing, and the use of toothpicks and additional use of fluoride.

\section{Abbreviations}

ACT: Acceptance and Commitment Therapy; CBT: Cognitive Behaviour Therapy; ITT: Intention-to-treat; ns.: Non-significant; PP: Per protocol

\section{Acknowledgements}

The research coordinators, the head of clinics and the dental personnel who contributed to identifying eligible participants are gratefully acknowledged. Licensed psychologist and ACT specialist Celia Young's role as an adviser is also gratefully acknowledged.

\section{Funding}

The study was supported by grants from The Health Care Subcommittee, Region Västra Götaland, Sweden.

\section{Availability of data and materials}

The data sets generated and/or analysed during the current study are not publicly available due to Regional Ethical Review Board regulations, but are available from the corresponding author on reasonable request.

Authors' contributions

$\mathrm{MH}$ and UW planned the study, HW and $\mathrm{JH}$ prepared the data set, and $\mathrm{MH}$ is responsible for the analysis of data. All authors are responsible for drafting the manuscript and have read and approved the final manuscript.

\section{Ethics approval and consent to participate}

Ethical consent to perform the study was obtained from the Regional Ethical Review Board, Gothenburg, Reg. no. 840-12. All participants gave their informed written consent to participate.

Consent for publication

Not applicable.

\section{Competing interests}

The authors declare that they have no competing interests.

\section{Publisher's Note}

Springer Nature remains neutral with regard to jurisdictional claims in published maps and institutional affiliations.

Received: 2 February 2018 Accepted: 24 September 2018

Published online: 03 October 2018

\section{References}

1. WHO. Sugars and dental caries. In: Technical information note. WHO/NMH/ $\mathrm{NHD} / 1712 ; 2017$.

2. Petersen PE. The world Oral health report 2003: continuous improvement of oral health in the 21 st century--the approach of the WHO global Oral health Programme. Community Dent Oral Epidemiol. 2003:31(Suppl 1):3-23.

3. Petersen PE. Oral health. In: International encyclopedia of public health. San Diego (CA): Academic Press; 2008. p. 677-85.

4. Guarnizo-Herreno CC, Watt RG, Pikhart H, Sheiham A, Tsakos G. Socioeconomic inequalities in oral health in different European welfare state regimes. J Epidemiol Community Health. 2013;67:728-35. 
5. Guarnizo-Herreno CC, Watt RG, Pikhart H, Sheiham A, Tsakos G. Inequalities in oral impacts and welfare regimes: analysis of 21 European countries. Community Dent Oral Epidemiol. 2014;42:517-25.

6. Sabbah W, Tsakos G, Chandola T, Sheiham A, Watt RG. Social gradients in oral and general health. J Dent Res. 2007;86:992-6.

7. Werner H, Hakeberg M, Dahlstrom L, Eriksson M, Sjogren P, Strandell A, et al. Psychological interventions for poor Oral health: a systematic review. J Dent Res. 2016;95:506-14.

8. Newton JT, Asimakopoulou K. Managing oral hygiene as a risk factor for periodontal disease: a systematic review of psychological approaches to behaviour change for improved plaque control in periodontal management. J Clin Periodontol. 2015;42 Suppl 16:S36-46.

9. Al Rawahi SH, Asimakopoulou K, Newton JT. Theory based interventions for caries related sugar intake in adults: systematic review. BMC Psychol. 2017;5:25.

10. Noar SM, Zimmerman RS. Health behavior theory and cumulative knowledge regarding health behaviors: are we moving in the right direction? Health Educ Res. 2005;20:275-90.

11. Bartholomew LK, Mullen PD. Five roles for using theory and evidence in the design and testing of behavior change interventions. J Public Health Dent 2011;71(Suppl 1):S20-33.

12. Hayes SC, Strosahl KD, Wilson KG. Acceptance and committment therapy. New York: Guilford; 1999.

13. Powers MB, MB ZVSV, Emmelkamp PM. Acceptance and commitment therapy: a meta-analytic review. Psychother Psychosom. 2009;78:73-80.

14. Ruiz FJ. Acceptance and commitment therapy versus traditional cognitive behavioral therapy: a systematic review and meta-analysis of current empirical evidence. Int J Psychol Psychol Ther. 2012;12:333-57.

15. Hayes SC, Luoma JB, Bond FW, Masuda A, Lillis J. Acceptance and commitment therapy: model, processes and outcomes. Behav Res Ther. 2006:44:1-25.

16. Pocock SJ. Clinical trials. Chichester: Wiley; 1983.

17. Moher D, Hopewell S, Schulz KF, Montori V, Gotzsche PC, Devereaux PJ et al. CONSORT 2010 explanation and elaboration: updated guidelines for reporting parallel group randomised trials. BMJ. 2010;340:c869.

18. Pitts NB, Fyffe HE. The effect of varying diagnostic thresholds upon clinical caries data for a low prevalence group. J Dent Res. 1988;67:592-6.

19. Strosahl K, Robinson P, Gustavsson T. Brief interventions for radical change. New Harbinger: Oakland; 2012

20. Robinson PJ, Gould DA, Strosahl KD. Real behavior change in primary care: improving patient outcomes and increasing job satisfaction. New Harbinger: Oakland, CA; 2010.

21. Borrelli B. The assessment, monitoring, and enhancement of treatment fidelity in public health clinical trials. J Public Health Dent. 2011; 71(Suppl 1):S52-63.

22. Pallant J. SPSS Survival manual. 5th ed. Berkshire, England: McGraw-Hill; 2013.

23. Cohen JW. Statistical power analysis for the behavioral sciences. Hillsdale, NJ: Lawrence Erlbaum Associates; 1988.

24. Renz A, Ide M, Newton T, Robinson PG, Smith D. Psychological interventions to improve adherence to oral hygiene instructions in adults with periodontal diseases. Cochrane Database Syst Rev. 2007;18:CD005097.

25. Jonsson B, Lindberg P, Oscarson N, Ohrn K. Improved compliance and selfcare in patients with periodontitis--a randomized control trial. Int J Dent Hyg. 2006:4:77-83

26. Jonsson B, Ohrn K, Oscarson N, Lindberg P. The effectiveness of an individually tailored oral health educational programme on oral hygiene behaviour in patients with periodontal disease: a blinded randomizedcontrolled clinical trial (one-year follow-up). J Clin Periodontol. 2009;36: 1025-34.

27. Kakudate N, Morita M, Sugai M, Kawanami M. Systematic cognitive behavioral approach for oral hygiene instruction: a short-term study. Patient Educ Couns. 2009;74:191-6.

28. Godard A, Dufour T, Jeanne S. Application of self-regulation theory and motivational interview for improving oral hygiene: a randomized controlled trial. J Clin Periodontol. 2011:38:1099-105.

29. Brand VS, Bray KK, MacNeill S, Catley D, Williams K. Impact of single-session motivational interviewing on clinical outcomes following periodontal maintenance therapy. Int J Dent Hyg. 2013;11:134-41.

30. Stenman J, Lundgren J, Wennstrom JL, Ericsson JS, Abrahamsson KH. A single session of motivational interviewing as an additive means to improve adherence in periodontal infection control: a randomized controlled trial. J Clin Periodontol. 2012;39:947-54.
31. Tedesco LA, Keffer MA, Davis EL, Christersson LA. Effect of a social cognitive intervention on oral health status, behavior reports, and cognitions. J Periodontol. 1992;63:567-75.

32. Little SJ, Hollis JF, Stevens VJ, Mount K, Mullooly JP, Johnson BD. Effective group behavioral intervention for older periodontal patients. J Periodontal Res. 1997;32:315-25.

33. Frank RG. Primary care psychology. Washington DC: American Psychological Association; 2003

34. Lundgren J, Wide Boman U. Multimodal cognitive behavioural treatment. In Öst L-G, Skaret E, editors. Cognitive behaviour therapy for dental phobia and anxiety. West Sussex UK: Wiley-Blackwell; 2013. p. 109-18.

35. Kani E, Asimakopoulou K, Daly B, Hare J, Lewis J, Scambler S, et al. Characteristics of patients attending for cognitive behavioural therapy at one UK specialist unit for dental phobia and outcomes of treatment. Br Dent J. 2015:219:501-6.

36. Agdal ML, Raadal M, Ost LG, Skaret E. Quality-of-life before and after cognitive behavioral therapy (CBT) in patients with intra-oral injection phobia. Acta Odontol Scand. 2012;70:463-70.

37. Ramseier CA, Suvan JE. Behaviour change counselling for tobacco use cessation and promotion of healthy lifestyles: a systematic review. J Clin Periodontol. 2015;42 Suppl 16:S47-58.

38. Fanshawe TR, Halliwell W, Lindson N, Aveyard P, Livingstone-Banks J, Hartmann-Boyce J. Tobacco cessation interventions for young people. Cochrane Database Syst Rev. 2017;11:CD003289.
Ready to submit your research? Choose BMC and benefit from:

- fast, convenient online submission

- thorough peer review by experienced researchers in your field

- rapid publication on acceptance

- support for research data, including large and complex data types

- gold Open Access which fosters wider collaboration and increased citations

- maximum visibility for your research: over $100 \mathrm{M}$ website views per year

At $\mathrm{BMC}$, research is always in progress.

Learn more biomedcentral.com/submissions 